\title{
Serum Creatinine in Normal Sudanese Athlets and Soldiers
}

\author{
Nagah A. A. Mohamed ${ }^{1, *}$, Jumana M. ELhafiz ${ }^{2}$, Faroug B.M.Ahmed ${ }^{3}$ \\ ${ }^{1}$ Sudan University of science and technology \\ ${ }^{2}$ Sudan Ministry of health \\ ${ }^{3}$ Department of biochemistry, Faculty of Science and Technology, Shendi University \\ *Corresponding author: nagahabdelwahab@hotmail.com,dr.nagah@sustech.edu
}

Received June 04, 2014; Revised June 19, 2014; Accepted September 10, 2014

\begin{abstract}
Plasma levels of creatinine are related to the relative muscles mass, the rate of creatine turnover and the renal function. It has been accepted for years that the plasma level of creatinine is relatively un affected by the diet. However, some reports have indicated that the protein content of the diet may be indeed affect the plasma level of creatinine if it affects the individuals muscles mass. This a primary study that aims to detect rough estimation of serum creatinine in normal Sudanese athletes and soldiers. In addition to detect weather creatinine level is associated by gender, age, daily working hours or occupation of study population. Hundred (49 soldiers: 51 athletes) normal individuals from Khartoum state invited to take a role in this study (75 male: 25 female) their age was between 1444 years. At $\mathrm{P} \leq 0.05$ this study showed no significant differences ( $\mathrm{P} 0.772$ ) in creatinine concentration with mean \pm SD $(1.07 \pm 0.11,1.12 \pm 0.17 \mathrm{mg} / \mathrm{dl})$ for soldiers and athletes respectively. There was no signifigant difference in creatinine concentration either between both sexes male: female (1.1 $\pm 0.16: 1.1 \pm 0.12)$, or between different occupations soldiers: athletes $(1.07 \pm 0.11: 1.12 \pm 0.17)$. But there was significant difference (Chi 0.027) in creatinine concentration $(0.5,1.0,1.5)$ for $(14-24,25-34,35-44)$ age groups respectively. Serum creatinine was not associated with daily working hours (ANOVA 0.18 ) with creatinine values $(1.16,1.01,1.12,1.13,0.8)$ for weekly working hours $(\leq 20,21-40,41-60,61-80,81-100)$. Serum creatinine is not much different depending on the gender, individual occupation or daily working hours, weather they were soldiers or athletes, but it is influenced by age progress. Sudanese soldiers and Athletes creatinine level fell within the normal international range. Research with large data collection is recommended for establishment and localization normal range of biochemical parameters for sport medicine in Sudan.
\end{abstract}

Keywords: soldiers athletes, serum creatinine, muscles

Cite This Article: Nagah A. A. Mohamed, Jumana M. ELhafiz, and Faroug B.M.Ahmed, "Serum Creatinine in Normal Sudanese Athlets and Soldiers.” American Journal of Clinical Medicine Research, vol. 2, no. 4 (2014): 84-86. doi: 10.12691/ajcmr-2-4-5.

\section{Introduction}

Exercise physiology is concern with the description and explanation of the changes that occur in human body during single or repeated bouts of muscular exercise. The chemical and physical changes which takes place during muscular exercise, trigger numerous responses in all systems of the body, to respond to increased demand of energy and oxygen and deal with the increased production of heat and waste products.(Concise, et al., 1993).

The study of muscular exercise has enabled athletes to attain better standard at different sports and made it possible to increase the efficiency of manual workers to do more work with less energy cost. (Ganong, 1999).

Certain changes occur in the function of various organs when exercise is repeated over period of time. The magnitude of the change depends on many factors, the most important being the density and frequency of exercise. Age and heredity may also play a role. The nature of the change depend on the type of exercise, the muscles used and the previous training of the individuals. To attain a training level, the intensity of exercise must be at least $60 \%$ (maximum exercise capacity) or $60 \%$ of maximum heart rate. Concise, et al., 1993).

The basic fuel that can supply substrates for oxidation by muscles are glucose, locally stored glycogen and free fatty acids or to lesser extend derived from triglycerol depots within the muscles. When oxidation energy is insufficient, muscles make use of anaerobic metabolism to obtain energy. There are two path ways first from phosphocreatine the second route is from glycogen or glucose with formation of lactate.(Vasudervan, et al., 2011).

Creatinine is formed in the muscles from creatine phosphate by irreversible, non enzymatic dehydration and loss of phosphate. The 24-hours urinary excretion of creatinine is proportionate to muscle mass produced is related to muscles mass (Murray, et al., 2007). Each day, $1-2 \%$ of muscle creatine is converted to creatinine. Men tend to have higher levels of creatinine than women 
because, in general, they have a greater mass of skeletal muscle. Increased dietary intake of creatine or eating a lot of meat can increase daily creatinine excretion (Taylor, 1989).

Kidneys are responsible for eliminating creatinine from the body when you urinate. When creatinine levels increase, your kidney function may be impaired (Roth, 2012). Determination of (creatinine clearance), from measurement of creatinine concentration in both a 24 hour urine collection and a serum specimen, provides a clinically useful estimate of GFR. Excretion rates of creatinine (quantity in urine/day) do not change in renal disease (Clark, 2000).

Factors which alter the creatinine level are old age, renal diseases (gloeruloepheritis, renal failure and urinary obstruction) and medicines such as amphotericin and captoril. Where, as factors reducing serum creatinine are low muscle mass, malnutrition and medicines as thiazide and vancomycin (Vasudevan, 2011).

Muscle mass gain can affect creatinine kinetics, leading to underestimation of the urinary biomarker-to-creatinine ratio (Tonomura, et al., 2013). In liver metabolism, the interpretation of serum aminotransferases concentration in athletes should consider the release of aspartate aminotransferase (AST) from muscle and of alanine aminotransferase (ALT) mainly from the liver. Muscle metabolism parameters such as creatine kinase (CK) are typically increased after exercise (Banfi, et al., 2012).

Serum creatinine concentration in athletes is related to body mass index. Prediction of glomerular filtration rate in athletes by means of creatinine-based equations is questionable because of discrepancies among formulae, owing to the particular anthropometric characteristics of athletes (high body mass index) (Fabbro, 2009). Certain changes occur in the function of various organs when exercise repeats over a period of time. Age and heredity also play a role. The primary effect occur in the skeletal muscles (Banfi, et al., 2009).

To the best of our knowledge there is lack of information regarding creatinine level in Sudanese individuals. So this study aims to detect rough estimation of serum creatinine in normal Sudanese athletes and soldiers. In addition to detect weather creatinine level is associated by gender, age, daily working hours or occupation of study population

\section{Material and methods}

\subsection{Subject}

Hundred (49 soldiers: 51 athletes) apparently absence of medical illness as sub stained by medical history and physical examination. None had weight fluctuation more than $2 \mathrm{~kg}$ during the last six months prior to testing and lived most of their lives in Sudan. Individuals mainly from Khartoum state invited to take a role in this study (75 male: 25 female) their age was between 14-44 years.

\subsection{Blood Samples}

Five $\mathrm{ml}$ of blood samples were collected. Serum separated by centrifuging blood for 10 minutes at 3000RPM. Then, decanted into $5 \mathrm{ml}$ plain plastic tube, labeled with name, date of collection and identification number of the volunteer participating in this study. Creatinine is estimates by using Cresent commercial kit under instructions of the manufacturer. The absorbance was read at $490 \mathrm{~nm}$ using Jenway colorimeter.

The biochemical tests were conducted in clinical chemistry laboratory, Faculty of medical laboratory sciences, Sudan University of science and technology. The precision and accuracy of all methods used in this study were checked each time a batch was analyzed by including commercially prepared control sera.

\subsection{Ethical Consideration}

An informed consent, aims and benefits of this study were explained to the participants

\subsection{Data Analysis}

The data was analyzed using Statistical Package for Social Sciences (SPSS), Windows version16, 1997 SPSS, Inc, Chicago, IL, and USA.

Mean, SD calculated independentent T -test, ANOVA and chi tests were used.

\section{Results}

At $\mathrm{P} \leq 0.05$ this study showed no significant differences ( $P$ 0.772) in creatinine concentration with mean \pm SD $(1.07 \pm 0.11,1.12 \pm 0.17 \mathrm{mg} / \mathrm{dl})$ for soldiers and athletes respectively. There was no signifigant difference in creatinine concentration either between both sexes male: female (1.1 \pm 0.16 : $1.1 \pm 0.12)$, or between different occupations soldiers: athletes $(1.07 \pm 0.11: 1.12 \pm 0.17)$. But there was significant difference (Chi 0.027) in creatinine concentration $(0.5,1.0,1.5)$ for $(14-24,25-34,35-44)$ age groups respectively. Serum creatinine was not associated with daily working hours (ANOVA 0.18 ) with creatinine values $(1.16,1.01$, $1.12,1.13,0.8)$ for weekly working hours $(\leq 20,21-40$, 41- 60, $61-80,81-100)$.

Table 1. Serum Creatinine level for both sexes

\begin{tabular}{|l|l|l|l|}
\multicolumn{4}{|c}{ Table 1. Serum Creatinine level for both sexes } \\
\cline { 1 - 2 } Gender & Mean $\pm \mathrm{SD}(\mathrm{mg} / \mathrm{dl})$ & Chi test value & Significance \\
\hline Male & $1.1 \pm 0.16$ & \multirow{2}{*}{0.669} & Not significant \\
\cline { 1 - 2 } & $1.1 \pm 0.12$ & & \\
\hline
\end{tabular}

Table 2. Serum Creatinine level in solders and athletes

\begin{tabular}{|l|l|l|l|}
\hline Occupation & Mean \pm SD $(\mathbf{m g} / \mathbf{d l})$ & P value & Significance \\
\hline Soldiers & $1.07 \pm 0.11$ & \multirow{2}{*}{0.772} & Not significant \\
\hline Athletes & $1.12 \pm 0.17$ & & \\
\hline
\end{tabular}

Table 3. Creatinine concentration in different age group of study participants

\begin{tabular}{|c|c|c|c|c|}
\hline Age years & $\mathbf{1 4} \mathbf{- 2 4}$ & $\mathbf{2 5} \mathbf{- 3 4}$ & $\mathbf{3 5} \mathbf{- 4 4}$ & Chi test \\
\hline Creatinine (mg/dl) & 0.5 & 1.0 & 1.5 & 0.027 \\
\hline
\end{tabular}

Table 4. Creatinine level against working hours

\begin{tabular}{|c|c|c|c|c|c|c|}
\hline Working hours per week & $\leq \mathbf{2 0}$ & $\mathbf{2 1 - 4 0}$ & $\mathbf{4 1 - 6 0}$ & $\mathbf{6 1}-\mathbf{8 0}$ & $\mathbf{8 1 - 1 0 0}$ & Anova value \\
\hline Creatinine mg/dl & 1.16 & 1.01 & 1.12 & 1.13 & 0.8 & 0.18 \\
\hline
\end{tabular}




\section{Discussion}

Muscles mass increase as result of training as more protein synthesized and less protein breakdown occur. Due to increase skeletal muscles mass in athletes' women creatinine concentration was the same in female as male. No significant differences found between the athletes and soldiers despite their hard physical activity. This finding in the same line as those reported finding by Banfi et al (2012) who reported Physical exercise induces adaptations in metabolism considered beneficial for health. Athletic performance is linked to adaptations, training, and correct nutrition in individuals with genetic traits that can facilitate such adaptations. Intense and continuous exercise, training, and competitions, however, can induce changes in the serum concentrations of numerous laboratory parameters. On the other hand it disagreed with Tonomura,et al (2013) who said Both plasma creatinine and excretion of urinary creatinine showed increases with muscle mass gain in rats, in which the alterations of urinary biomarker-to-creatinine (UBCR) were lowered. Muscle mass gain can affect creatinine kinetics, leading to underestimation of UBCR.

The study showed that the age had a clear effect on the rate of serum creatinine where the study showed a significant difference between the studied groups $(\mathrm{p}<0,05)$. This was attributed to the age progress which causes cells destruction, then lead to increasing of serum creatinine and this is identical with Vasudevan (2011).

Females was expected to have lower level of creatinine than males, but this test proved that it was the same.The reason might be that those females practice certain types of exercise regularly that helped them in raising their serum creatinine to be as same as male.

Sudanese soldiers and Athletes creatinine level fell within the normal international range. Large data collection is recommended for localization of creatinine level in Sudanese people in addition to more sophisticated research study should be conducted for nutritional assessments, anthropometric measurements and other biochemical studies in Sudanese soldiers and athletes.
Health promotion programs should be introduced by the authorities.

\section{Conclusion}

Sudanese soldiers and Athletes creatinine level fell within the normal international range. Serum creatinine is not much different depending on the gender, individual occupation or daily working hours, weather they were soldiers or athletes, but it is influenced by age progress.

\section{References}

[1] Allen P.J. (2012).Creatine metabolism and psychiatric disorders: Dose creatine supplementation have therapeutic value?. Neurosci Biobehav Re 36 (5): 1442-62.

[2] Banfi G., Colombin A., Lombadig G. and Lubkowska A. (2012). Metabolic markers in sports medicine. Adv Clin Chem, 2012; 56: 1-54.

[3] Clark J. (2000). Laboratory medicine and pathology. Laboratory Evaluation of Renal Function: Creatinine Clearance as an Estimate of GFR -www.easybib search. March, 9, 2000.

[4] Fabbro M.,. and Lippi G. (2009). Serum creatinine concentration and creatinine-based estimation of glomerular filtration rate in athletes. Sport Med.; 39(4):331-7.

[5] Ganon,W.F.(1999).Review of medical physiology, $19^{\text {th }}$ edn. Appleton and Lange, San Francisco, California.

[6] Gross J, L., de Azevedo M.J., Silveiro S.P., Canani L.H., Caramori M.L. and Zelmanovitz T. (2005). "Diabetic nephropathy: diagnosis, prevention, and treatment". Diabetes Care 28 (1): 16476.

[7] Murray K.R,Granner K.D. and Rodwell W.V. (2007). Harpper’s illustrated biochemistry, $27^{\text {th }}$ edition, Mc Graw Hill Lange, international edition, p: 274.

[8] Roth E. (2012). Creatinine Blood Test. Health line. August 20(5) 13-20.

[9] Taylor E. H. (1989). Clinical Chemistry. New York: John Wiley and Sons. pp. 4, 58-62.

[10] Tonomura Y, Morikawa Y, Takagi S, Torii M, Matsubara M. (2013) Underestimation of urinary biomarker-to-creatinine ratio resulting from age-related gain in muscle mass in rats. Toxicology Jan 7; 303: 169-76.

[11] Vasudervan DM., Sreekumari and Vaidyanathan K. (2011). Textbook of biochemistry for medical students, $6^{\text {th }}$, Jaypee brothers medical publishers (p) LTD. P: 320-21. 